\title{
Synergistic effects of glycated chitosan with high-intensity focused ultrasound on suppression of metastases in a syngeneic breast tumor model
}

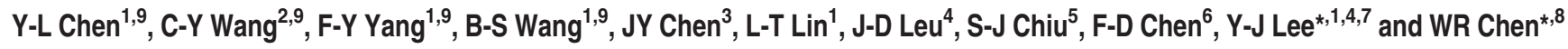

Stimulation of the host immune system is crucial in cancer treatment. In particular, nonspecific immunotherapies, when combined with other traditional therapies such as radiation and chemotherapy, may induce immunity against primary and metastatic tumors. In this study, we demonstrate that a novel, non-toxic immunoadjuvant, glycated chitosan (GC), decreases the motility and invasion of mammalian breast cancer cells in vitro and in vivo. Lung metastatic ratios were reduced in $4 \mathrm{~T} 1$ tumorbearing mice when intratumoral GC injection was combined with local high-intensity focused ultrasound (HIFU) treatment. We postulate that this treatment modality stimulates the host immune system to combat cancer cells, as macrophage accumulation in tumor lesions was detected after GC-HIFU treatment. In addition, plasma collected from GC-HIFU-treated tumor-bearing mice exhibited tumor-specific cytotoxicity. We also investigated the effect of GC on epithelial-mesenchymal transition-related markers. Our results showed that GC decreased the expression of Twist-1 and Slug, proto-oncogenes commonly implicated in metastasis. Epithelial-cadherin, which is regulated by these genes, was also upregulated. Taken together, our current data suggest that GC alone can reduce cancer cell motility and invasion, whereas GC-HIFU treatment can induce immune responses to suppress tumor metastasis in vivo.

Cell Death and Disease (2014) 5, e1178; doi:10.1038/cddis.2014.159; published online 17 April 2014

Subject Category: Cancer

Metastasis is a major cause of cancer treatment failure and mortality. Metastasis to distant organ sites requires enhanced cell motility and invasion. ${ }^{1,2}$ An ideal cancer treatment modality should suppress and eradicate metastasis; however, effective methods are yet to be developed. ${ }^{3}$

Immunotherapy holds great promise in cancer treatment, as it stimulates the host immune system to target cancer cell surface markers to achieve therapeutic effects. These anticancer immunological responses can be augmented with cytokines or interleukins. ${ }^{4}$ Thus, the use of immunoadjuvants in conjunction with direct cancer treatment can stimulate systemic, tumor-specific immunity, ${ }^{5}$ as is the case with the synergistic use of immunoadjuvants in optical or laser-based therapies. ${ }^{6-8}$

Glycated chitosan (GC), a novel immunoadjuvant, is derived from chitosan, which is a linear polysaccharide composed of D-glucosamine and $\mathrm{N}$-acetyl-D-glucosamine through (1-4) linkage. ${ }^{9} \mathrm{GC}$ contains $\mathrm{D}(+)$ galactose molecules and is water soluble, allowing for a wide variety of biomedical applications. ${ }^{10}$ When combined with laser irradiation, GC is effective in treating human breast cancer and melanomas. ${ }^{11-15}$ In addition, preclinical studies ${ }^{10-12,16}$ and preliminary clinical trials ${ }^{17}$ have shown that GC, when combined with phototherapy, induces an immunological response against both treated primary tumors and untreated distant metastases. However, it remains unclear whether GC can directly influence tumor cell behavior, such as migration and invasion.

High-intensity focused ultrasound (HIFU) is a newly developed method of noninvasively ablating tumor cells. ${ }^{18,19}$ Although its mechanism remains to be fully determined, preliminary studies show that the acoustic cavitation and the absorbed heat induced by the interaction between ultrasound pulses and microbubbles may puncture cells and cause tissue necrosis and subsequent immune response. ${ }^{20,21}$ In addition, HIFU-induced cellular responses can modulate systemic

\footnotetext{
${ }^{1}$ Department of Biomedical Imaging and Radiological Sciences, National Yang-Ming University, Taipei, Taiwan; ${ }^{2}$ Department of Radiotherapy, Cheng Hsin General Hospital, Taipei, Taiwan; ${ }^{3}$ Case Western Reserve University School of Medicine, Cleveland, OH 44106, USA; ${ }^{4}$ Division of Radiation Oncology, Taipei City Hospital RenAi Branch, Taipei, Taiwan; ${ }^{5}$ Department of Life Sciences, TzuChi University, Hualien, Taiwan; ${ }^{6}$ Graduate Institute of Biotechnology, Chinese Culture University, Taipei 11114, Taiwan; ${ }^{7}$ Biophotonics and Molecular Imaging Research Center (BMIRC), National Yang-Ming University, Taipei 112, Taiwan and ${ }^{8}$ Biophotonics Research Laboratory, Department of Engineering and Physics, University of Central Oklahoma, Edmond, OK 73034, USA

${ }^{*}$ Corresponding author: Y-J Lee, Biomedical Imaging and Radiological Sciences, National Yang-Ming University, 155, Linong Street Section 2, Beitou District, Taipei 112, Taiwan. Tel: + 88622826 7189; Fax: + 88622820 1095; E-mail: yjlee2 @ym.edu.tw

or WR Chen, Department of Engineering and Physics, University of Central Oklahoma, Edmond, OK 73034, USA. Tel: +1 4059745147 ; Fax: + 1405 974 3824 ; E-mail: wchen@uco.edu

${ }^{9}$ These authors contributed equally to this work.

Keywords: immunoadjuvant; high-intensity focused ultrasound; lung metastasis; breast cancer; epithelial-mesenchymal transition

Abbreviations: GC, glycated chitosan; HIFU, high-intensity focused ultrasound; EMT, epithelial-mesenchymal transition; mRFP, monomeric red fluorescence protein; E-cadherin, epithelial-cadherin; MTT, 3-[4,5-dimethylthiazol-2-yl]-2,5diphenylterazoliumbromide; PBS, phosphate-buffered saline; ELISA, enzyme-linked immunosorbent assay; EDTA, ethylenediaminetetraacetic acid

Received 02.1.14; revised 13.3.14; accepted 13.3.14; Edited by A Stephanou
} 
antitumor immunity. ${ }^{19,22}$ Furthermore, HIFU-mediated tissue destruction via cavitation can be augmented by intravenous administration of microbubbles.

We investigated the in vitro effect of GC on the motility and invasion of murine 4T1 breast cancer cells and human MDAMB-231 breast cancer cells. We also studied the in vivo effect of GC and microbubbles/HIFU on these cell lines with bioluminescent imaging of tumor progression in mice using a multicistronic reporter gene system. Furthermore, we analyzed the influence of GC on epithelial-mesenchymal transition (EMT) of cancer cells as well as on GC-HIFU induced antimetastatic immunological responses.

\section{Results}

Reduction of motility and invasion of breast cancer cells by GC. We used a wound healing assay to measure the migration rate of $4 \mathrm{~T} 1$ cells with and without $\mathrm{GC}$ treatment $(100 \mu \mathrm{g} / \mathrm{ml}$ of $\mathrm{GC}$ for $24 \mathrm{~h})$. GC significantly reduced the motility of $4 \mathrm{~T} 1$ cells compared with the untreated control for up to $6 \mathrm{~h}$ (Figure 1a). Cell migration rates were quantified using ImageJ software (National Institutes of Health, Bethesda, MD, USA) (Figure 1b).

Subsequently, we performed an in vitro invasion assay using Matrigel-coated transwells, which showed that GC also reduced the invasion of $4 \mathrm{~T} 1$ cells compared with untreated controls (Figures $1 \mathrm{c}$ and d). Reduced cell migration and invasion were also detected in human MDA-MB-231 breast cancer cells treated by GC (Figures 1e and f). The dose and treatment duration have been described in the Materials and Methods. The 3-[4,5-dimethylthiazol-2-yl]-2,5diphenylterazoliumbromide (MTT) assays showed that viability of both 4T1 cells and MDA-MB-231 cells was not significantly affected by $\mathrm{GC}$ up to a concentration of $250 \mu \mathrm{g} / \mathrm{ml}$ (Figure $1 \mathrm{~g}$ ), suggesting that operational levels of GC were non-cytotoxic.

Transfection of multicistronic reporter genes to 4T1 cells for the purpose of in vivo imaging. A new 4T1_PB3R line of cells was created as described in the Materials and Methods. These cells exhibited high luciferase activity as measured by luminescent assay (Figure 2a). The expression of monomeric red fluorescence protein (mRFP) in 4T1_PB3R cells was pronounced compared with that of parental 4T1 cell, as visualized under fluorescence microscope (Figure $2 \mathrm{~b}$ ). The growth rate (Figure $2 \mathrm{c}$ ) and invasion rate (Figure 2d) of 4T1_PB3R cells were the same as that of the parent $4 \mathrm{~T} 1$ cells. In addition, the viability of 4T1_PB3R cells was not affected by GC, even at a concentration up to $250 \mu \mathrm{g} / \mathrm{ml}$ (Figure 2e). These results indicate that it is appropriate to use the 4T1_PB3R cell line as a surrogate for monitoring the behavior of $4 \mathrm{~T} 1$ tumor cells in vivo via reporter gene imaging.

Suppression of tumor metastasis by GC-HIFU treatment. 4T1_PB3R cells were subcutaneously implanted into $\mathrm{Balb} / \mathrm{C}$ mice. After tumors reached a size of $100 \mathrm{~mm}^{3}$, tumorbearing mice were injected with microbubbles via tail vein and treated with HIFU, followed by intratumoral injection of GC (Figure 3a, see Materials and Methods). Tumor-bearing mice were also treated with GC only or HIFU only. The bioluminescent images showed that in untreated mice, tumors metastasized to various locations within 28 days of implantation (Figure 3b). Tumor metastasis was also detectable in mice treated with GC only or HIFU only, but was suppressed in GC-HIFU-treated mice (Figure 3b). Bioluminescent signals from outside the primary tumor in mice of different experimental groups were detected and compared with that of the untreated control group. It appears that GC-HIFU-treated mice showed reduced bioluminescent signals in non-primary tumor sites, suggesting inhibited metastasis (Figure 3c).

Although 4T1 cells metastasize to various primary organs, including the lung, liver, bone and brain, we focused on lung metastases in this study because they occurred in all untreated mice (data not shown). We collected lung tissue from tumor-bearing mice for histological analysis 4 weeks after each treatment. Lung metastases were noticeably reduced in mice treated by GC-HIFU compared with all other groups (Figure $3 \mathrm{~d}$ ). The size and the number of nodules in affected lungs were visualized and measured (Supplementary Figure 1). These findings strongly suggest that GC and HIFU have a synergistic effect on the suppression of lung metastasis of breast cancer.

Accumulation of macrophages in tumors after GC-HIFU treatment. We measured macrophage activity to demonstrate the immunomodulatory effect of GC-HIFU treatment. Tumor sections were collected 2 weeks after GC, HIFU or GC-HIFU treatment. Immunohistochemical (IHC) staining analysis was used to detect antibodies against the F4/80 protein, a macrophage marker, on each tumor section. There was an accumulation of $\mathrm{F} 4 / 80$ markers in primary tumors treated by GC-HIFU (Figure $4 \mathrm{a}$ ). The results of IHC were also quantified by counting the number of F4/80-stained cells in tumor sections (Figure 4b). Therefore, GC-HIFU treatment may enhance the innate immune response. Whether this effect leads to modulation of metastasis is unknown and needs to be further investigated.

Cytotoxic effects of plasma from GC-HIFU-treated tumor-bearing mice. Blood plasma was extracted from tumor-bearing mice 2 weeks after GC, HIFU or GC-HIFU treatment. The plasma was diluted $(1: 10)$ in culture medium and added to cultured 4T1_PB3R cells in a 96-well plate. Cytotoxicity was then determined using the MTT assay (Figure 5a). Figure 5b shows that plasma obtained from GC-HIFU-treated mice markedly inhibited the viability of 4T1_PB3R cells compared with plasma from any other treatment group (Figure 5b). On the contrary, the viability of non-tumorigenic NIH-3T3 fibroblasts was not affected by any of these treatments (Figure 5b). These results suggest that GC-HIFU treatment may induce tumor-specific immunity.

Effects of GC on EMT-related markers. Epithelialmesenchymal transition (EMT) is an important mechanism for promoting metastasis. According to previous reports, Twist-1, Snail, and Slug are upregulated, whereas epithelial cadherin (E-cadherin) is downregulated during EMT. ${ }^{23-25} \mathrm{We}$ compared the protein levels of Twist-1, Snail, Slug, and 
a
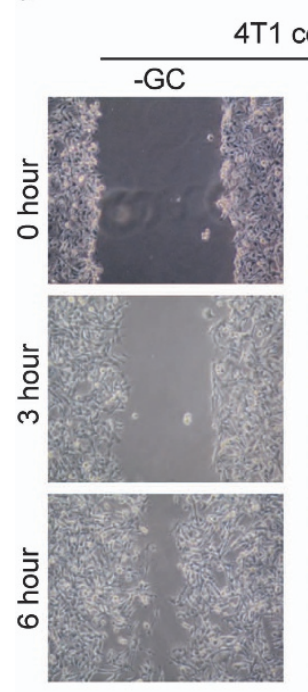

b

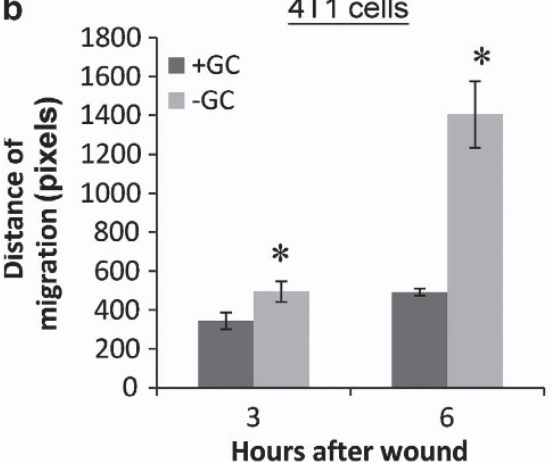

C

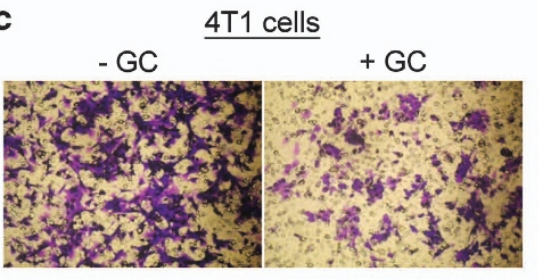

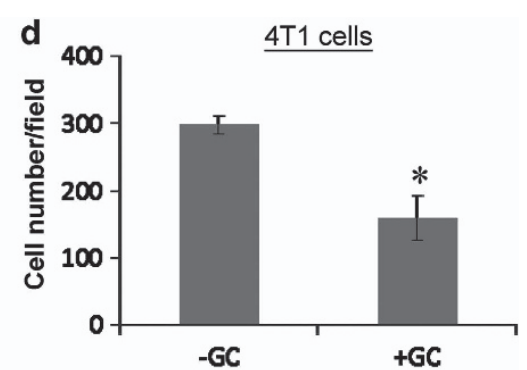

e MDA-MB-231 cells

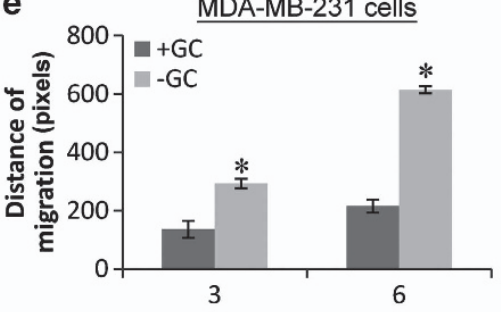

Hours after wound

f

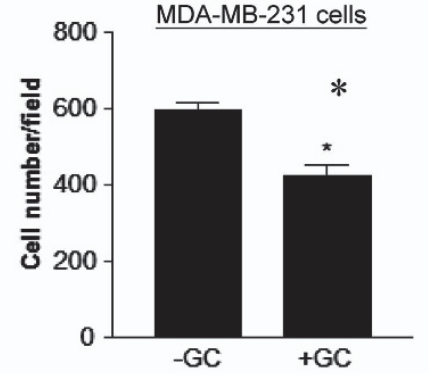

g

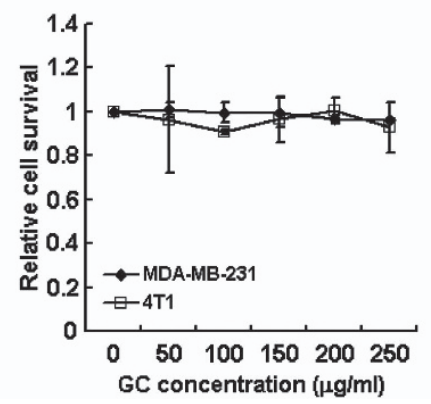

Figure 1 Suppression of migration and invasion of breast cancer cells by GC in vitro. (a) Wound healing assay of $4 \mathrm{~T} 1 \mathrm{cells}$ before and after $\mathrm{GC}$ treatment ( $100 \mu \mathrm{g} / \mathrm{ml}$ ) of up to $6 \mathrm{~h}$. (b) Distance of cell migration obtained using ImageJ software. (c) Visualization of $4 \mathrm{~T} 1$ cells transpassing transwells coated with Matrigel before and after GC treatment. (d) Quantification of transpassed 4T1 cell count using photos of four randomly selected fields. (e) Quantification of GC-mediated suppression of migration of human MDA-MB231 breast cancer cells using wound healing assay. (f) Quantification of GC-mediated suppression of invasion of human MDA-MB-231 breast cancer cells using in vitro invasion assay. (g) Cytotoxic effects of GC $(50-250 \mu \mathrm{g} / \mathrm{ml})$ on $4 \mathrm{T1}$ cells and MDA-MB-231 cells using MTT assay. ${ }^{*} P<0.05$ in each subfigure

E-cadherin in 4T1_PB3R cells before and after GC treatment using western blot analysis. Twist-1 levels were slightly reduced after 10 and $100 \mu \mathrm{g} / \mathrm{ml}$ of $\mathrm{GC}$ treatment for $24 \mathrm{~h}$; further suppression of Twist-1 and Slug was found up to $48 \mathrm{~h}$, but the expression of Snail was not significantly reduced by GC treatment (Figure 6a). The level of E-cadherin was also upregulated by GC; however, lower concentrations of GC showed a greater impact than higher concentrations (Figure 6a). Densitometric quantification of immunoblots further confirmed this observation (Figure 6b). These results suggest that GC may influence the expression of EMTrelated makers, at least in part.

\section{Discussion}

GC is an effective nonspecific immunoadjuvant for cancer treatment in combination with noninvasive laser photothermal therapy. ${ }^{6,8,10,17,26-28}$ HIFU can increase the permeability of blood vessels and mediate antitumor immune responses. ${ }^{29-31}$ Thus, we investigated the effect of GC, HIFU, and GC-HIFU on the motility, invasion, and metastatic potential of the 4T1 tumor line. Our wound healing assay and cell migration test show that GC alone reduces cell motility and invasion, despite its lack of direct toxicity toward 4T1 and other cells (Figure 2). 


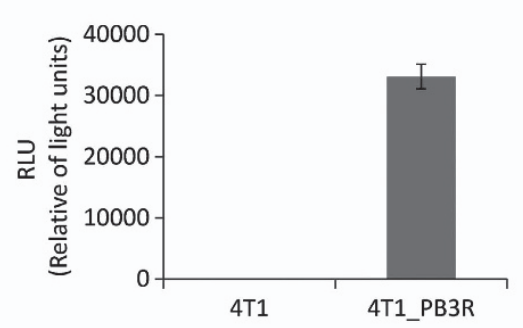

b
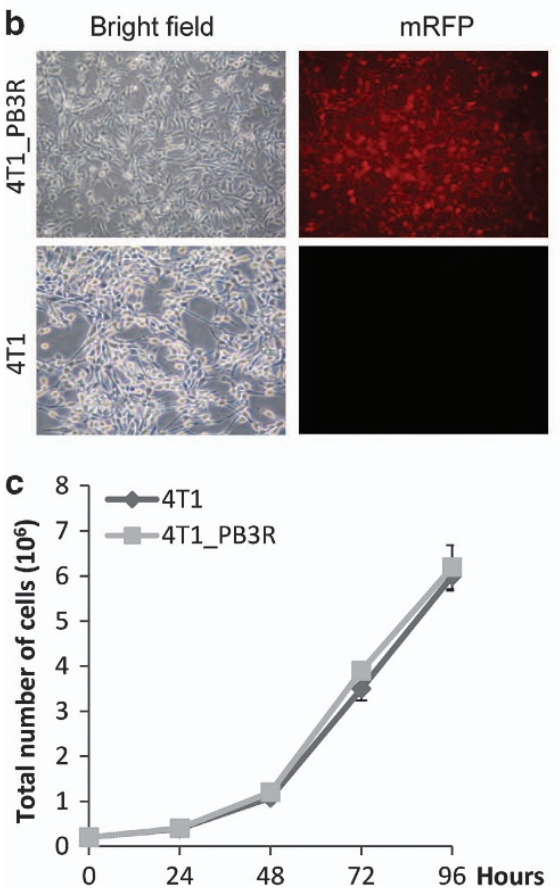

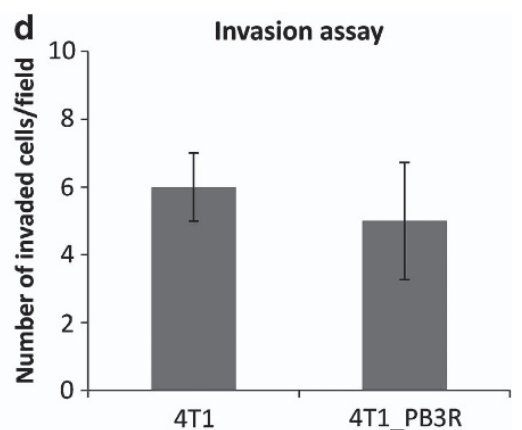

e

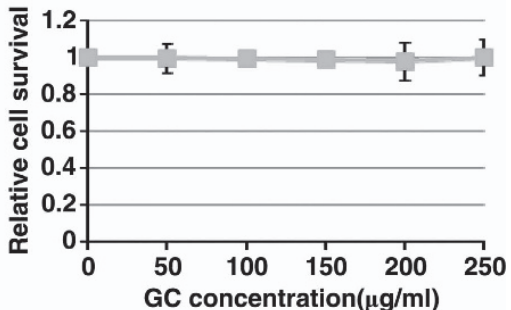

Figure 2 Establishment of a stable 4T1_PB3R cell line for in vivo imaging of tumor progression. (a) Luc2 activity in 4T1_PB3R cells determined using luciferase gene reporter assay. (b) Expression of mRFP in 4T1_PB3R cells but not in parental 4T1 cells was detected using fluorescence microscopy. (c) Comparison of growth rates between 4T1_PB3R cells and 4T1 cells. (d) In vitro invasion of 4T1_PB3R cells and 4T1 cells. (e) Cell viability using MTT assay after treatment with GC of different concentrations

To monitor effectively tumor progression in vivo, we created a new 4T1_PB3R cell line. These cells emit both bioluminescent signals (by luciferase expression) and fluorescent signals (by mRFP expression) that can be used for cell tracking in vivo and in vitro, as shown in Figures $2 a$ and $b$, while maintaining the properties of their parent 4T1 line, such as growth (Figure 2c), migration (Figure 2d), and GC treatment response (Figure 2e).

Our bioluminescent imaging results indicate that GC-HIFU treatment can reduce the metastatic ratio of 4T1_PB3R breast cancer in mice. It is noteworthy that neither treatment by HIFU nor GC alone had any significant effect on the primary tumor or lung metastases (Figures $3 \mathrm{~b}$ and $\mathrm{c}$ ). A recent report proposed that low pressure-pulsed focused ultrasound with microbubbles could promote antitumor immunological responses in a xenograft CT-26 colon tumor animal model. ${ }^{29}$ Additionally, pulsatile HIFU has been reported to be beneficial for drug delivery in tumor treatment. ${ }^{32}$ Whether this mode can directly stimulate immune responses or enhance immune responses in the presence of an immunostimulator (GC in our case) is unknown. We thus decided to use pulsatile HIFU in combination with GC in our in vivo studies. Although our results did not completely agree with previous findings, a different tumor model, ultrasound apparatus, and treatment parameters, may have led to this discrepancy. Because continuous HIFU provides better thermal therapeutic effects on tumor, ${ }^{33}$ it may be used in combination with GC in the future as a comparator to this study. Future investigations could correlate HIFU parameters and tumor growth, as well as address the auxiliary role of GC on pulsatile HIFU delivery. Furthermore, HIFU delivery parameters should be optimized to maximize primary tumor eradication.

In this study, the GC-HIFU combination was successful in inducing accumulation and activation of macrophages in treated tumors compared with treatment by GC or HIFU alone (Figure 4). Although this study demonstrated that GC-HIFU could induce significant immune responses in tumors, it does not exclude the possibility that intratumoral injection using needles may cause enough physical damage to induce immune responses. The use of intratumoral injection of GC 
a

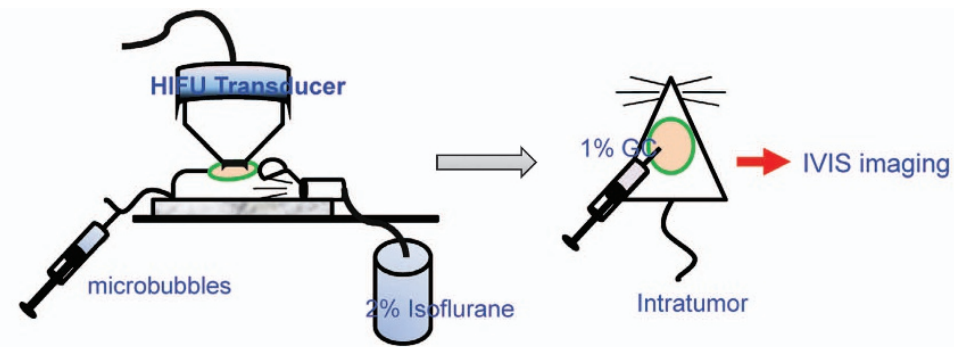

b

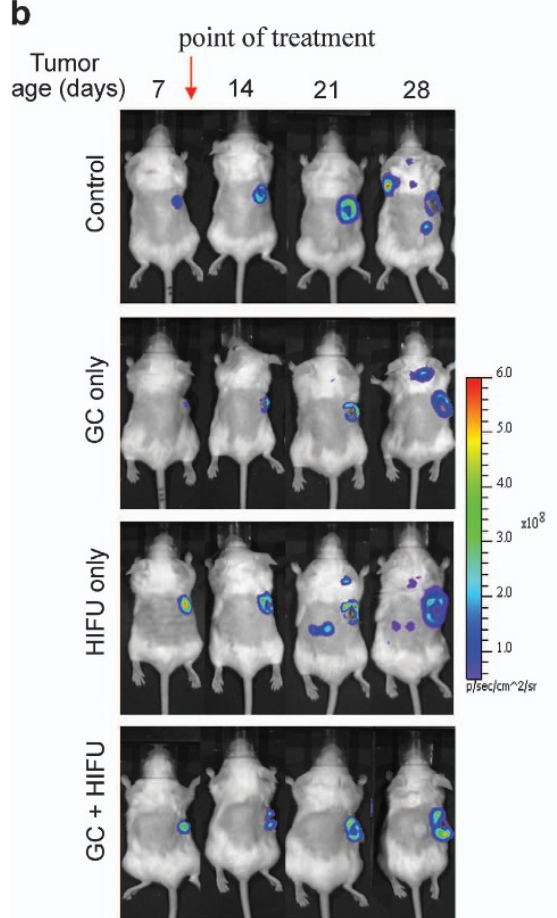

C
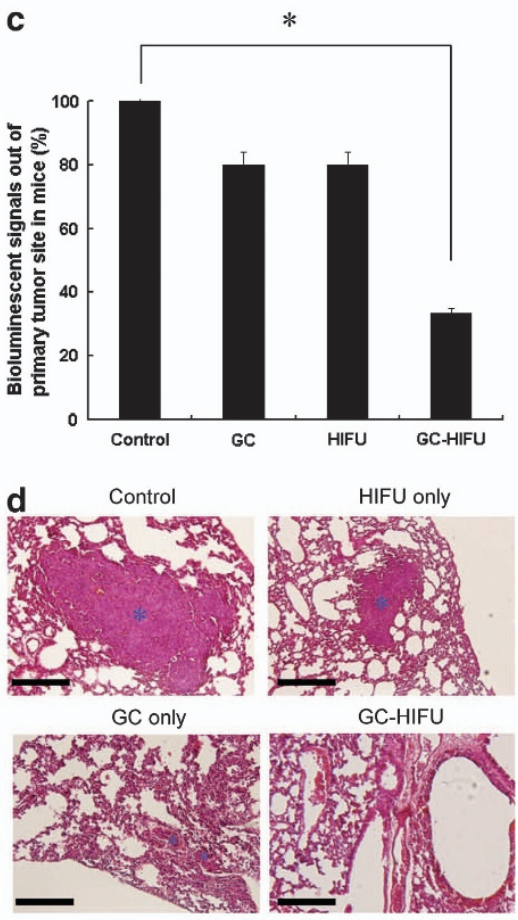

Figure 3 Suppression of lung metastasis of 4T1_PB3R cells in female Balb/c mice by GC-HIFU treatment. (a) Schematics of GC-HIFU treatment. (b) Progression of 4T1_PB3R tumors in mice under different treatments, determined by IVIS imaging system ( $N=6$ for each experimental group). (c) Bioluminescent signals from non-primary tumor sites among different experimental groups, in comparison with that of untreated control group, according to the IVIS data. ${ }^{*} P<0.05$. (d) Tumor metastasis in lung sections detected using hematoxylin and eosin staining (marked with asterisks). The scale bar of each picture is $200 \mu \mathrm{m}$

is based on previous studies; ${ }^{13,16}$ however, other administration routes may be investigated to avoid potent side effects.

We have demonstrated that combined GC-HIFU treatment exhibits a synergistic effect in reducing metastases (Figures $3 \mathrm{~b}$ and $\mathrm{c}$ ). However, it is unclear whether GC-HIFU-induced accumulation of macrophages in primary tumors is directly related to reduced lung metastasis in vivo. Because tumor growth at the primary site was not suppressed (Figure 3b), macrophage accumulation may be insufficient to inhibit tumor proliferation. Most interesting is our discovery that plasma extracted from mice treated by GC-HIFU reduces the viability of cultured 4T1 cells but not NIH-3T3 fibroblasts (Figure 5b), suggesting a tumor-specific immunity induced by GC-HIFU. The antimetastatic effects of GC-HIFU may be associated with an increase in cytokine release. However, we used enzyme-linked immunosorbent assay (ELISA) to analyze the level change of tumor necrosis factor- $\alpha$ (TNF- $\alpha$ ), and showed no significant difference among all experimental groups (data not shown). Therefore, use of cytokine-based protein array analysis in the future will help us understand which cytokines are implicated in GC-HIFU-mediated systemic immune responses.

We found that GC displays remarkable efficacy in reducing mobility and invasion of $4 \mathrm{~T} 1$ cells and MDA-MB-231 cells, despite the fact that GC is non-toxic to tumor cells. ${ }^{11,12}$ Thus, we postulate that this GC-mediated suppression is not caused by direct cytotoxicity. GC suppressed the Twist-1 and the Slug transcription factors, and induced E-cadherin expression, but did not suppress the expression of the Snail transcription factor that is also influential in EMT. ${ }^{34}$ Twist-1 and Snail have been reported to be essential for the maintenance of late EMT and initiation of EMT, respectively. ${ }^{35}$ Because $4 \mathrm{~T} 1$ breast cancer cells are highly metastatic cells, we speculate that this cell type has entered late EMT so that the expression of Twist-1, but not Snail, was more treatment-susceptible. However, further studies are required to better understand the discrepancy between Snail and other EMT-related marker expression after GC treatment. Although more EMT-related markers should be examined, our current data suggest that GC at least partially inhibits EMT. Moreover, whether GC 
a

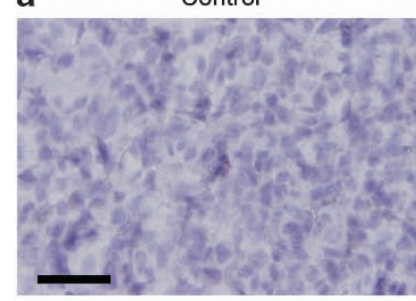

GC only
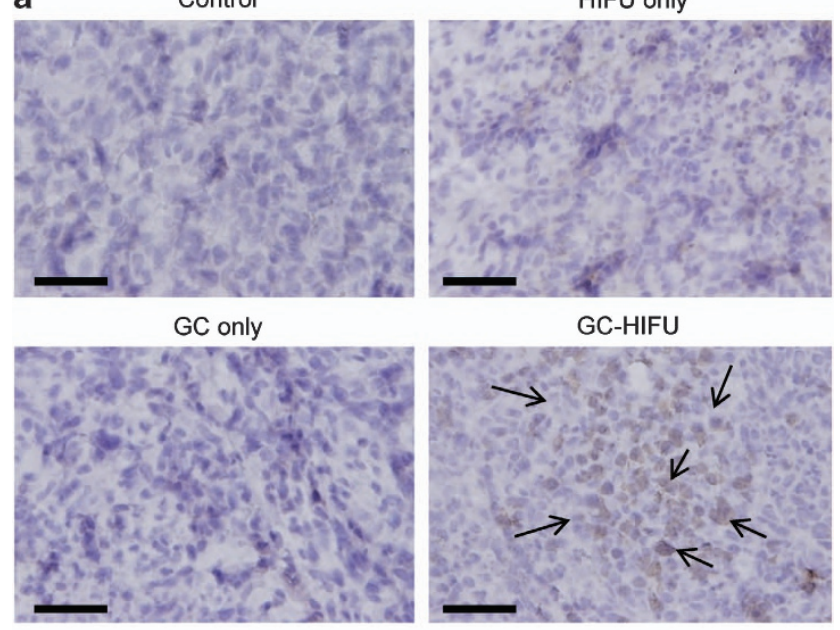

GC-HIFU

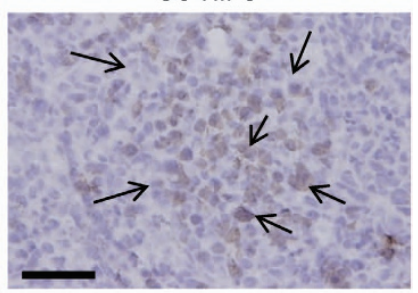

b

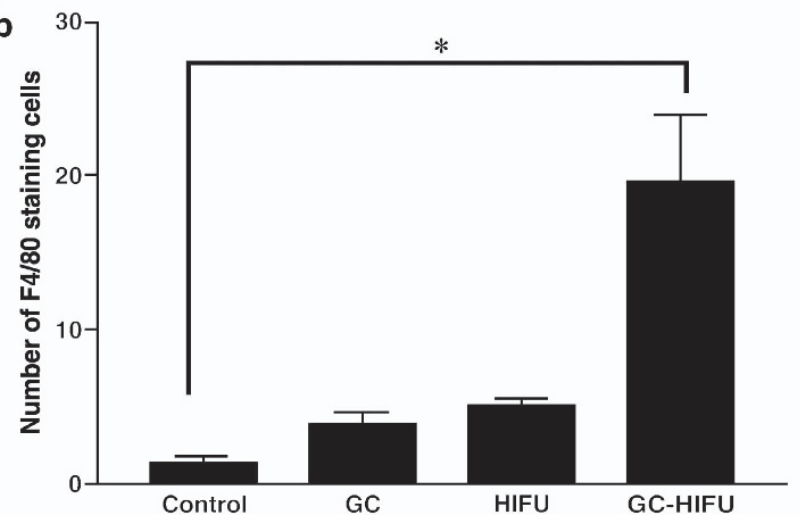

Figure 4 Accumulation of macrophages in primary tumors after different treatments using IHC staining. (a) Detection of F4/80 murine macrophage markers. The arrows indicate macrophage marker F4/80 in tumor lesions. The scale bar of each picture is $50 \mu \mathrm{m}$. (b) Quantification and comparison of F4/80 markers in primary tumors in different treatment groups. The data represent the mean \pm S.D. from three randomly selected tissue sections. ${ }^{*} P<0.05$

influences the change of other migration- and invasion-related molecules, such as matrix metalloproteinases and tissue inhibitor of metalloproteinases family proteins would be of great interest for further investigation. A microarray assay may be helpful to better understand the underlying mechanisms of GC-mediated inhibition of tumor motility and invasion.

Although GC exhibited antimigratory effects on cultured cells, it appeared that GC alone was insufficient to suppress tumor metastasis in animal models. The discrepancies between in vitro and in vivo effects have been reported in various studies. ${ }^{36-39}$ As shown in our earlier studies, GC may be effective in stimulating a systemic immune response through its interactions with tumor cells at treatment sites. ${ }^{12,13,15}$ GC's antitumor effect appeared to be mediated by activation of immune cells in vivo, rather than direct inhibition of tumor cell migration, although it is difficult to measure the inhibition effect on tumor cells in vivo. Furthermore, GC was administered about 7 days after tumor cell seeding in mice. Because the antimigratory effects of GC on cell culture were only investigated in an hourly manner (see the wound healing assay), it seems impossible to reflect a

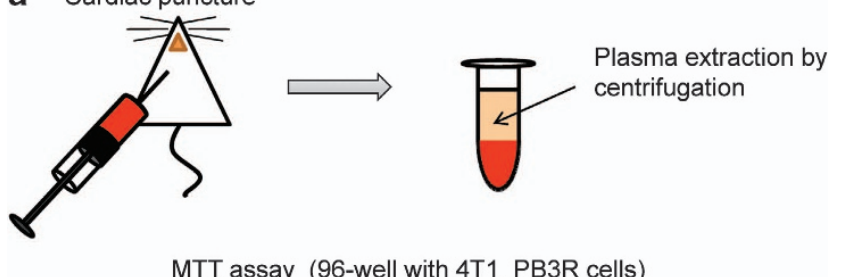

MTT assay (96-well with 4T1_PB3R cells)

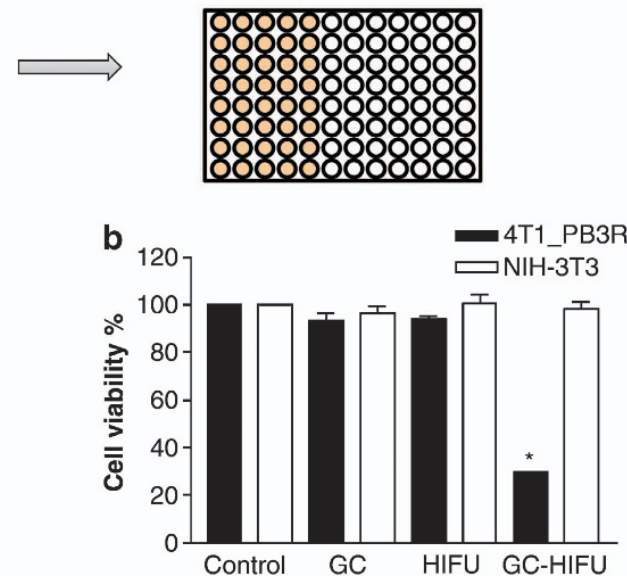

Figure 5 Cytotoxic effects of blood plasma obtained from GC-HIFU-treated tumor-bearing mice. (a) Schematic for plasma extraction and test of plasma cytotoxic effects in cultured 4T1_PB3R cells. Plasma was extracted 2 weeks after GC and/or HIFU treatment. (b) Cell viability using MTT assay after the incubation of tumor cells with serum from mice after different treatments. GC-HIFU treatment induced significant cytotoxic effects on 4T1_PB3R cells but not on NIH-3T3 fibroblasts compared with untreated controls. ${ }^{*} P<0.05$

completely the in vivo results of GC treatment because tumor progression was monitored for several weeks after early treatment of GC.

In summary, our results demonstrate that GC, as an immunoadjuvant, is able to reduce the migration of 4T1 breast cancer cells. This effect is likely associated with suppression of EMT-related molecules. The GC-HIFUinduced immune response was demonstrated through the accumulation of macrophages at tumor lesions as well as potent plasma immunity. Combining GC and HIFU results in a synergistic effect on the reduction of the lung metastatic ratio of $4 \mathrm{~T} 1$ breast cancer cells in vivo, which may be related to HIFU-mediated direct tumor destruction and GC-mediated antitumor immunity. This combination may become the foundation for a feasible cancer treatment modality, particularly for metastatic cancers.

\section{Materials and Methods}

Cell culture. 4T1 cells are triple-negative (lacking the expression of estrogen, progesterone, and Her2/neu receptors) murine breast carcinoma cells that closely mimic human breast cancer in both tumor growth and metastasis. These cells were cultured in RPMl1640 medium (Gibco; Invitrogen Inc., Carlsbad, CA, USA) with 10\% fetal bovine serum (HyClone; Thermo, Waltham, MA, USA), $1 \%$ penicillin-streptomycin solution $(100 \times)$ (Caisson Laboratories Inc., North Logan, UT, USA), and 1\% L-glutamine (200 mM) (Sigma-Aldrich Co., St. Louis, MO, USA). Human triple-negative MDA-MB-231 breast cancer cells ${ }^{40}$ and NIH-3T3 cells were maintained in Dulbecco's modified Eagle medium (Gibco; Invitrogen Inc.) with $10 \%$ fetal bovine serum, $1 \%$ penicillin-streptomycin solution, and $1 \%$ L-glutamine $(200 \mathrm{mM})$. Cells were maintained in an incubator containing $5 \%$ $\mathrm{CO}_{2}$ at $37^{\circ} \mathrm{C}$ and were passaged every 2 days. 
a
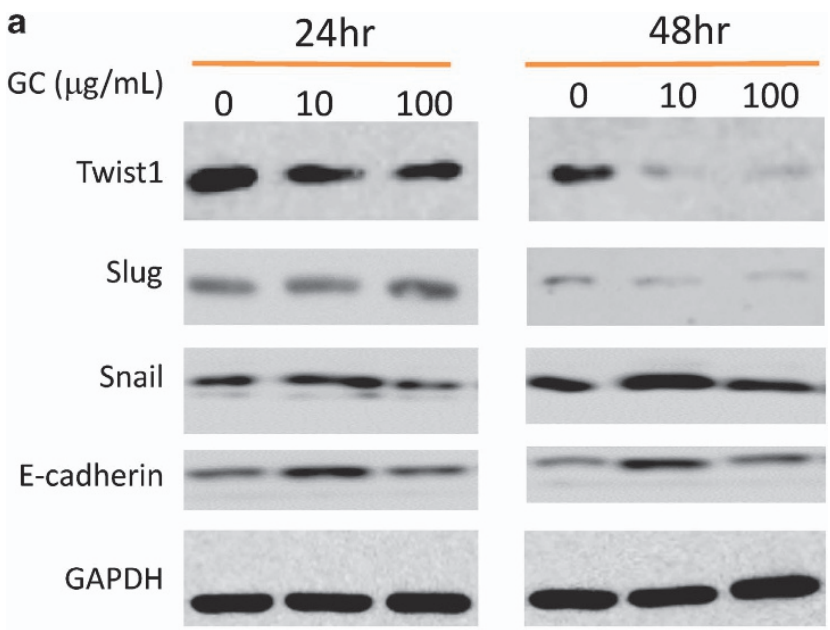

b

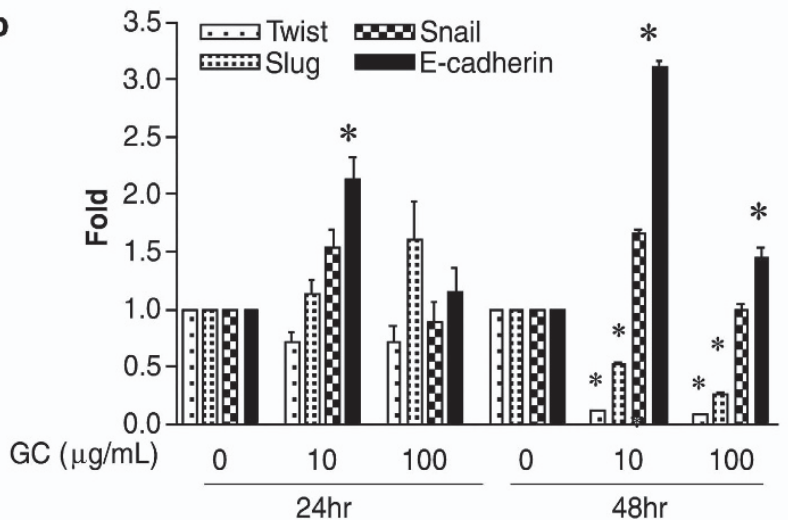

Figure 6 Effects of GC on the expression of EMT-related markers. (a) Western blot analysis for Twist-1, Snail, and E-cadherin, after 4T1 cells were treated with GC at indicated doses with different treatment durations. (b) Quantification of band intensity in each blot using densitometry. Each data point represents mean \pm S.D. of three independent experiments. ${ }^{*} P$-value $<0.05$ for Twist-1, E-cadherin, and Slug, compared with untreated controls

Transfection of multicistronic reporter genes to 4T1 cells to create a new 4T1_PB3R cell line. We previously established a stable murine 4T1-PB-2R/PBase breast cancer cell line containing monomeric red fluorescent protein (mRFP) and herpes simplex virus type 1-thymidine kinase reporter genes using the piggyBac transposon system for in vivo tumor imaging. ${ }^{41}$ We modified this system by adding a firefly luciferase (Iuc2) gene to the original dual-cistronic PB-2R construct to create a new PB-3R construct for two reasons. First, we wanted to overcome the low sensitivity of mRFP for optical imaging. Second, luc2 has a high sensitivity for bioluminescent imaging of metastases. 4T1 cells were stably transfected with this multicistronic reporter gene system to create a new 4T1_PB3R cell line. The PB-3R-puro plasmid was constructed from PB-2R plasmid that was provided by Dr. Yu Kang and Dr. Congjian Xu. The 4T1_PB3R cells were cultured in RPMI medium as described above.

GC treatment. GC $(10 \mathrm{mg} / \mathrm{ml}$, dissolved in deionized distilled water) was prepared as described previously. ${ }^{10} \mathrm{GC}$ was stored at $4{ }^{\circ} \mathrm{C}$ and was transferred to room temperature before usage. The dosage of GC administration was dependent on the experimental purposes as described below.

Wound healing assay. $4 \mathrm{~T} 1$ cells $\left(2 \times 10^{5}\right.$ cells per well) were seeded in a 6 -well plate. After cells reached $80 \%$ confluence, $100 \mu \mathrm{g} / \mathrm{ml}$ of GC was added to the wells for $24 \mathrm{~h}$. Phosphate-buffered saline (PBS) of the same volume was added to the cells in the control groups. The wounds were then incised using a $200 \mu \mathrm{l}$ Pipetman tip in three random positions in each well. Detached cells were washed with PBS and fresh medium was then added. Wound healing was visualized at 3 and $6 \mathrm{~h}$ under a light microscope to confirm gap width consistency.
The wound healing rate was determined by visualizing cell migration to the scraped area using light microscopy, and images were acquired and analyzed using ImageJ software (version 1.46).

In vitro invasion assay. Matrigel (BD Biosciences, San Jose, $\mathrm{CA}$, USA) was mixed with $25 \mu \mathrm{l}$ serum-free medium at a ratio of $1: 4$, added into transwells (24 Well Millicell $8.0 \mu \mathrm{m}$; Millipore Co., Billerica, MA, USA), and then incubated at $37^{\circ} \mathrm{C}$ overnight. Cultured 4T1 cells grown in exponential phase were treated with $100 \mu \mathrm{g} /$ $\mathrm{ml}$ of GC or left untreated for $24 \mathrm{~h}$ and then trypsinized. Cells $\left(1 \times 10^{5}\right)$ were then mixed with $200 \mu \mathrm{l}$ serum-free medium and added into each transwell. Each transwell was then placed into a 24-well plate containing $400 \mu \mathrm{l}$ normal medium in each well. After $24 \mathrm{~h}$ of incubation, each transwell was washed with $1 \times$ phosphate-buffered saline (PBS) and fixed in 4\% paraformaldehyde (Sigma-Aldrich Co., St. Louis, MO, USA) for $10 \mathrm{~min}$. The transwells were then incubated in $1.25 \%$ crystal violet solution (Sigma-Aldrich $\mathrm{Co}_{0}$ ) for $2 \mathrm{~min}$. After PBS destaining, the membranes embedded in the transwells were cut and placed onto slides for microscopic visualization. Crystal violet-stained cells on the membrane were then counted.

Cell viability assay. $4 \mathrm{~T} 1$ cells were seeded in a $96-$ well plate (800 cells per well) with GC of various concentrations $(0,50,100,150,200$, and $250 \mu \mathrm{g} / \mu \mathrm{l}$, and incubated at $37^{\circ} \mathrm{C}$ for 4 days. After removal of the supernatant, $1 \mathrm{mg} / \mathrm{ml} \mathrm{MTT}$ solution (Sigma-Aldrich Co.) was mixed with serum-free medium and added to each well. The cells were then incubated at $37^{\circ} \mathrm{C}$ for $3 \mathrm{~h}$. After removal of the MTT solution, $100-\mu$ l dimethyl sulfoxide was added to dissolve crystals. The plate was then placed in an ELISA reader (ELISA plate reader; Bio-Tek Instruments, Winooski, VT, USA) and cell viability was determined by light absorption at $570 \mathrm{~nm}$.

Animal tumor model. 4T1 and 4T1_PB3R tumor cells $\left(1 \times 10^{6}\right.$ in $100 \mu \mathrm{l}$ serum-free medium) were subcutaneously injected into the upper backs of $\sim 4$ - to 5-week-old female Balb/c mice ( $N=6$ for each experimental group) (National Laboratory Animal Center, Taipei, Taiwan). Animal protocols were reviewed and approved by the Institutional Animal Care and Use Committee (IACUC) of National Yang-Ming University (Taipei, Taiwan; approval number: 1011103).

When the tumors became palpable, their dimensions were measured via caliper two times a week. We estimated tumor volume with the equation volume $=$ length $(\mathrm{mm}) \times$ width $(\mathrm{mm})^{2} / 2{ }^{41}$

GC and HIFU treatment of animal tumors. Before HIFU treatment, the skin overlying the tumor was epilated and covered with ultrasound transmission gel (Pharmaceutical Innovations, Newark, NJ, USA). The ultrasound contrast agent used in this procedure contained phospholipid-coated microbubbles with a mean diameter of $2.5 \mu \mathrm{m}$, at a concentration of $1 \times 10^{8}-5 \times 10^{8}$ bubbles per ml. The tumor-bearing mice were then injected with microbubbles $(300 \mu l / k g)$ via tail vein. HIFU was subsequently supplied using a single-element focused ultrasound transducer (A392S; Panametrics, Waltham, MA, USA) with a diameter of $38 \mathrm{~mm}$, a radius of curvature of $63.5 \mathrm{~mm}$, and a center frequency of $1 \mathrm{MHz}$. The transducer delivered HIFU at an acoustic power of $1.43 \mathrm{~W}$, a pulse repetition frequency of $1 \mathrm{~Hz}$, and a duty cycle of $5 \%$ for $2 \mathrm{~min}$. The setup for the transducer-driving system was the same as that of our previous work. ${ }^{42,43}$ After HIFU, a 100- $\mu$ l GC solution $(10 \mathrm{mg} / \mathrm{ml})$ was injected into the center and three additional random locations in each tumor using a $27 \mathrm{G}$ needle as described in previous reports, with slight modifications. ${ }^{16}$

In vivo bioluminescent imaging. 4T1_PB3R tumor cells expressing a firefly luciferase gene were subcutaneously injected into the upper backs of Balb/c mice. Each tumor-bearing mouse ( $N=6$ for each experimental group) then received an intraperitoneal injection of $150 \mathrm{mg} / \mathrm{kg}$ D-luciferin (Caliper Co., Hopkinton, MA, USA). After $15 \mathrm{~min}$, animals were anesthetized with $2 \%$ isofluorane, and luminescent signals were detected via IVIS50 system (Xenogen Co., Alameda, CA, USA). Region of interest tracings were drawn around each tumor site and luminescent signal was quantified by the number of photons detected per second.

IHC staining. Lung tissue samples were excised from three random tumorbearing mice after different treatments for 28 days and immediately fixed in $4 \%$ paraformaldehyde (Sigma-Aldrich Co.) overnight and then embedded with paraffin. Ten tissue sections (each $5 \mu \mathrm{m}$ ) were collected for analysis. They were deparaffinized using xylene immersion (Sigma-Aldrich Co.) for $30 \mathrm{~min}$, rehydrated for $5 \mathrm{~min}$ in 70,80 , or $90 \%$ ethanol, and then stained with hematoxylin and eosin. 
We also performed IHC analysis of macrophage markers in primary tumors. Tumor sections were treated with antigen retrieval solution $\left(100^{\circ} \mathrm{C} 2,2^{\prime}, 2^{\prime \prime}, 2^{\prime \prime \prime}\right.$ (ethane-1,2-diyldinitrilo)tetraacetic acid (EDTA), $\mathrm{pH}=8$ ) for $25 \mathrm{~min}$ and cooled on ice for $30 \mathrm{~min}$. The sections were subsequently blocked with $3 \% \mathrm{H}_{2} \mathrm{O}_{2}$ and protein blocking reagent (Biogenex Laboratories, San Ramon, CA, USA) in the dark and then incubated with anti-mouse F4/80 antibody (eBioscience Inc., San Diego, CA, USA) for $2 h$, followed by horseradish peroxidase (HRP)-conjugated secondary antibody for $1 \mathrm{~h}$. Finally, tissue sections were incubated with Liquid DAB + Substrate Chromogen System (Dako North America Inc., Carpinteria, VA, USA) until a brown color developed, and then counterstained with hematoxylin. The slides were visualized under light microscope, entire tissue sections were examined, and three random regions were selected and photographed using a digital camera.

Plasma extraction. Mice ( $N=6$ for each experimental group) were treated with GC and HIFU as described above and killed using cervical dislocation. Blood was immediately collected via cardiac puncture using a $26 \mathrm{G}$ needle and a syringe filled with $120 \mathrm{mg} / \mathrm{ml}$ EDTA. Extracted blood was centrifuged at 1000 r.p.m. at $4{ }^{\circ} \mathrm{C}$ for $10 \mathrm{~min}$. The supernatant was then collected and centrifuged two more times. The plasma was then sterilized with a $0.2 \mu \mathrm{m}$ Super Membrane Low Protein Binding filter (PALL Inc., Port Washington, NY, USA). Plasma was diluted (1:10) in culture medium and added into a 96-well plate seeded with 4T1 cells for MTT analysis.

Western blot analysis. 4T1 cells were incubated in GC solution at different concentrations $(0,10$, and $100 \mu \mathrm{g} / \mathrm{ml})$ for 24 and $48 \mathrm{~h}$. Protein was then extracted from cells using protein lysis buffer $(50 \mathrm{mM}$ Tris- $\mathrm{HCl}, 120 \mathrm{mM} \mathrm{NaCl}, 0.5 \% \mathrm{NP}-40)$ with $2 \%$ PMSF and quantified using the Bio-Rad Protein Assay (Bio-Rad Laboratories Inc., Hercules, CA, USA). Protein was mixed with sampling buffer (250 mM Tris- $\mathrm{HCl}$ (pH 6.8), 10\% sodium dodecyl sulfate (SDS), 30\% glycerol, $5 \%$ $\beta$-mercaptoethanol, $0.02 \%$ bromophenol blue), denatured with heating, and subjected to SDS-polyacrylamide gel electrophoresis Gel was electrotransferred to a nitrocellulose membrane (BioTraceTM NT; Pall, Port Washington, NY, USA) after electrophoresis, and the membrane was blocked with $4 \%$ milk in Tris-buffered saline with Tween-20 buffer (150 mM NaCl, $10 \mathrm{mM}$ Tris- $\mathrm{HCl}(\mathrm{pH} 8.0)$, and $0.1 \%$ Tween-20) for $2 \mathrm{~h}$. The membrane was incubated with primary antibody overnight, and followed by HRP-conjugated secondary antibody. The membrane was rinsed with Western lightning plus-ECL (Perkin-Elmer Inc., Waltham, MA, USA) and the chemoluminescent signals were detected using the LAS-4000 gel imaging system (GE Healthcare Inc., Wauwatosa, WI, USA). The band densities were quantified using ImageJ software (version 1.46). The primary antibodies used in this study included anti-Twist-1, anti-Slug, anti-Snail and anti-E-cadherin (kindly provided by Dr. Muh-Hwa Yang at National Yang-Ming University, Taipei, Taiwan). Antiglyceraldehyde 3-phosphate dehydrogenase antibodies were used as a control and were purchased from GeneTex Inc. (Irvine, CA, USA).

Statistical analysis. Experimental data were represented as the mean of three independent experiments \pm S.D. Data were analyzed with $t$-test or Mann-Whitney test (for animal experiments), with $P<0.05$ indicating statistical significance.

\section{Conflict of Interest}

The authors declare no conflict of interest.

Acknowledgements. We thank Dr. Yu Kang and Dr. Congjian Xu (Fudan University, Shanghai, China) for providing Act-PBase and PB-tk-mRFP plasmids for this work. We also thank Dr. Muh-Hwa Yang for providing antibodies for the EMT study. This research was supported in part by grants from the US National Institutes of Health (R21 EB0155091-01), the US Fulbright Scholar Program, and Immunophotonics Inc. This research was also supported by the united grant of Cheng-Hsin General Hospital and National Yang-Ming University (102F218C15), a grant from Cheng Hsin General Hospital (102-56), a grant from the Department of Health in Taipei City Government (10201-62-068) and the National Science Council of Taiwan (NSC102-2628-B-010-012-MY3), and a grant from the Ministry of Education, Aim for the Top University Plan, National Yang-Ming University.

1. Chambers AF, Groom AC, MacDonald IC. Dissemination and growth of cancer cells in metastatic sites. Nat Rev Cancer 2002; 2: 563-572.

2. Hainaut $P$, Plymoth $A$. Targeting the hallmarks of cancer: towards a rational approach to next-generation cancer therapy. Curr Opin Oncol 2013; 25: 50-51.
3. Wells A, Grahovac J, Wheeler S, Ma B, Lauffenburger D. Targeting tumor cell motility as a strategy against invasion and metastasis. Trends Pharmacol Sci 2013; 34: 283-289.

4. Vanneman M, Dranoff G. Combining immunotherapy and targeted therapies in cancer treatment. Nat Rev Cancer 2012; 12: 237-251.

5. Azuma I, Seya T. Development of immunoadjuvants for immunotherapy of cancer. Int Immunopharmacol 2001; 1: 1249-1259.

6. Chen WR, Singhal AK, Liu H, Nordquist RE. Antitumor immunity induced by laser immunotherapy and its adoptive transfer. Cancer Res 2001; 61: 459-461.

7. Ma X, Pan H, Yi J. Combination sonodynamic therapy with immunoadjuvant may be a promising new modality for cancer treatment. Med Hypothes 2009; 72: 418-420.

8. Chen WR, Liu H, Ritchey JW, Bartels KE, Lucroy MD, Nordquist RE. Effect of different components of laser immunotherapy in treatment of metastatic tumors in rats. Cancer Res 2002; 62: 4295-4299.

9. Kafetzopoulos D, Martinou A, Bouriotis V. Bioconversion of chitin to chitosan: purification and characterization of chitin deacetylase from Mucor rouxii. Proc Natl Acad Sci USA 1993; 90: 2564-2568.

10. Chen WR, Carubelli R, Liu H, Nordquist RE. Laser immunotherapy: a novel treatment modality for metastatic tumors. Mol Biotechnol 2003; 25: 37-44.

11. Song S, Zhou F, Nordquist RE, Carubelli R, Liu H, Chen WR. Glycated chitosan as a new non-toxic immunological stimulant. Immunopharmacol Immunotoxicol 2009; 31: 202-208.

12. Zhou F, Song S, Chen WR, Xing D. Immunostimulatory properties of glycated chitosan. J X-ray Sci Technol 2011; 19: 285-292.

13. Chen WR, Korbelik M, Bartels KE, Liu H, Sun J, Nordquist RE. Enhancement of laser cancer treatment by a chitosan-derived immunoadjuvant. Photochem Photobiol 2005; 81: 190-195.

14. Nordquist RE, Bishop SL, Ferguson H, Vaughan MB, Jose J, Kastl $\mathrm{K}$ et al. Immunohistochemical analysis of immune response in breast cancer and melanoma patients after laser immunotherapy. Proc. SPIE 7900, Biophotonics and Immune Responses VI, 790007; 23 February 2011, pp 790007-790010; doi:10.1117/12.874875.

15. Li X, Min M, Du N, Gu Y, Hode T, Naylor M et al. Chitin, chitosan, and glycated chitosan regulate immune responses: the novel adjuvants for cancer vaccine. Clin Dev Immunol 2013; 2013: 387023.

16. Zhou F, Wu S, Song S, Chen WR, Resasco DE, Xing D. Antitumor immunologically modified carbon nanotubes for photothermal therapy. Biomaterials 2012; 33: 3235-3242.

17. Li X, Ferrel GL, Guerra MC, Hode T, Lunn JA, Adalsteinsson 0 et al. Preliminary safety and efficacy results of laser immunotherapy for the treatment of metastatic breast cancer patients. Photochem Photobiol Sci 2011; 10: 817-821.

18. Hung BP. The effect of high intensity focused ultrasound ablation of human breast cancer. Surgery 2010; 147: 466-467.

19. Wu F, ter Haar G, Chen WR. High-intensity focused ultrasound ablation of breast cancer. Expert Rev Anticancer Ther 2007; 7: 823-831.

20. Haen SP, Pereira PL, Salih HR, Rammensee HG, Gouttefangeas C. More than just tumor destruction: immunomodulation by thermal ablation of cancer. Clin Dev Immunol 2011; 2011: 160250.

21. Xu J, Bigelow TA, Riesberg GM. Impact of preconditioning pulse on lesion formation during high-intensity focused ultrasound histotripsy. Ultrasound Med Biol 2012; 38: 1918-1929.

22. Hu Z, Yang XY, Liu Y, Morse MA, Lyerly HK, Clay TM et al. Release of endogenous danger signals from HIFU-treated tumor cells and their stimulatory effects on APCs. Biochem Biophys Res Commun 2005; 335: 124-131.

23. Zavadil J, Bottinger EP. TGF-beta and epithelial-to-mesenchymal transitions. Oncogene 2005; 24: 5764-5774.

24. Fendrich V, Waldmann J, Feldmann G, Schlosser K, König A, Ramaswamy A et al. Unique expression pattern of the EMT markers Snail, Twist and E-cadherin in benign and malignant parathyroid neoplasia. Eur J Endocrinol/Eur Fed Endocr Soc 2009; 160: 695-703.

25. Montserrat N, Gallardo A, Escuin D, Catasus L, Prat J, Gutiérrez-Avignó FJ et al. Repression of E-cadherin by SNAIL, ZEB1, and TWIST in invasive ductal carcinomas of the breast: a cooperative effort? Hum Pathol 2011; 42: 103-110.

26. Chen WR, Adams RL, Carubelli R, Nordquist RE. Laser-photosensitizer assisted immunotherapy: a novel modality for cancer treatment. Cancer Lett 1997; 115: 25-30.

27. Chen WR, Zhu WG, Dynlacht JR, Liu H, Nordquist RE. Long-term tumor resistance induced by laser photo-immunotherapy. Int J Cancer J 1999; 81: 808-812.

28. Chen WR, Jeong SW, Lucroy MD, Wolf RF, Howard EW, Liu H et al. Induced antitumor immunity against DMBA-4 metastatic mammary tumors in rats using laser immunotherapy. Int J Cancer 2003; 107: 1053-1057.

29. Liu HL, Hsieh HY, Lu LA, Kang CW, Wu MF, Lin CY. Low-pressure pulsed focused ultrasound with microbubbles promotes an anticancer immunological response. J TransI Med 2012; 10: 221.

30. Lu P, Zhu XQ, Xu ZL, Zhou Q, Zhang J, Wu F. Increased infiltration of activated tumorinfiltrating lymphocytes after high intensity focused ultrasound ablation of human breast cancer. Surgery 2009; 145: 286-293.

31. Wu F, Zhou L, Chen WR. Host antitumour immune responses to HIFU ablation. Int $J$ Hyperthermia 2007; 23: 165-171.

32. Yang FY, Lin GL, Horng SC, Chen RC. Prenatal exposure to diagnostic ultrasound impacts blood-brain barrier permeability in rats. Ultrasound Med Biol 2012; 38: 1051-1057.

33. Khokhlova TD, Hwang JH. HIFU for palliative treatment of pancreatic cancer. $J$ Gastrointest Oncol 2011; 2: 175-184.

34. Kang Y, Massague J. Epithelial-mesenchymal transitions: twist in development and metastasis. Cell 2004; 118: 277-279. 
35. Tran DD, Corsa CA, Biswas H, Aft RL, Longmore GD. Temporal and spatial cooperation of Snail1 and Twist1 during epithelial-mesenchymal transition predicts for human breast cancer recurrence. Mol Cancer Res 2011; 9: 1644-1657.

36. lacobelli S, Ranelletti FO, Longo P, Riccardi R, Mastrangelo R. Discrepancies between in vivo and in vitro effects of glucorticoids in myelomonocytic leukemic cells with steroid receptors. Cancer Res 1978; 38: 4257-4262.

37. Schug M, Stober R, Heise T, Mielke H, Gundert-Remy U, Godoy P et al. Pharmacokinetics explain in vivo/in vitro discrepancies of carcinogen-induced gene expression alterations in rat liver and cultivated hepatocytes. Arch Toxicol 2013; 87: 337-345.

38. Schurgers E, Kelchtermans H, Mitera T, Geboes L, Matthys P. Discrepancy between the in vitro and in vivo effects of murine mesenchymal stem cells on T-cell proliferation and collagen-induced arthritis. Arthritis Res Ther 2010; 12: R31.

39. Bove K, Lincoln DW, Tsan MF. Effect of resveratrol on growth of $4 \mathrm{~T} 1$ breast cancer cells in vitro and in vivo. Biochem Biophys Res Commun 2002; 291: 1001-1005.

40. Chavez KJ, Garimella SV, Lipkowitz S. Triple negative breast cancer cell lines: one tool in the search for better treatment of triple negative breast cancer. Breast Dis 2010; 32 $35-48$.

41. Chen YL, Wang SY, Liu RS, Wang HE, Chen JC, Chiou SH et al. Remnant living cells that escape cell loss in late-stage tumors exhibit cancer stem cell-like characteristics. Cell Death Dis 2012; 3: e399.
42. Yang FY, Wang HE, Lin GL, Teng MC, Lin HH, Wong TT et al. Micro-SPECT/CT-based pharmacokinetic analysis of ${ }^{99} \mathrm{mTC}$-diethylenetriaminepentaacetic acid in rats with bloodbrain barrier disruption induced by focused ultrasound. J Nucl Med 2011; 52: 478-484.

43. Yang FY, Wang HE, Liu RS, Teng MC, Li JJ, Lu M et al. Pharmacokinetic analysis of 111 in-labeled liposomal Doxorubicin in murine glioblastoma after blood-brain barrier disruption by focused ultrasound. PLoS One 2012; 7: e45468.

(c) $($ () $\ominus$ Cell Death and Disease is an open-access journal published by Nature Publishing Group. This work is licensed under a Creative Commons Attribution-NonCommercialNoDerivs 3.0 Unported License. The images or other third party material in this article are included in the article's Creative Commons license, unless indicated otherwise in the credit line; if the material is not included under the Creative Commons license, users will need to obtain permission from the license holder to reproduce the material. To view a copy of this license, visit http://creativecommons.org/licenses/ by-nc-nd/3.0/

Supplementary Information accompanies this paper on Cell Death and Disease website (http://www.nature.com/cddis) 